\title{
Off-Centered Stagnation Point Flow of a Couple Stress Fluid towards a Rotating Disk
}

\author{
Najeeb Alam Khan and Fatima Riaz \\ Department of Mathematical Sciences, University of Karachi, Karachi 75270, Pakistan \\ Correspondence should be addressed to Najeeb Alam Khan; njbalam@yahoo.com
}

Received 29 August 2013; Accepted 19 October 2013; Published 3 February 2014

Academic Editors: S. Torii and S. Zhang

Copyright ( 2014 N. A. Khan and F. Riaz. This is an open access article distributed under the Creative Commons Attribution License, which permits unrestricted use, distribution, and reproduction in any medium, provided the original work is properly cited.

An investigation has been made to study the off-centered stagnation flow of a couple stress fluid over a rotating disk. The model developed for the governing problem in the form of partial differential equations has been converted to ordinary differential equations with the use of suitable similarity transformation. The analytical approximation has been made with the most promising analytical approach, homotopy analysis method (HAM). The convergence region of the obtained solution is determined and plotted. The effects of couple stress and nondimensional parameters have been observed on the flows of couple stress fluid. Also comparison has been made with the Newtonian fluid as the special case of considered problem.

\section{Introduction}

The hydrodynamic flow of a viscous fluid due to the rotation of an infinite disk was the subject initially considered by Von Kármán [1] and has extensive applications in the fields of technology, engineering, industries, aerodynamics, and so forth. The work of Von Kármán has been extended to the immense directions by considering various forms of fluids, as well as diverse disk types [2-5]. The need of the century is to consider and investigate the non-Newtonian couple stress fluids which have wide real life applications in flow problems. The researchers are now paying adequate attention in this dimension and adding pioneer works in the literature in this regard. Stokes [6] was the first who developed the couple stress fluid theory which preserves couple stresses and body couples and represents the simplest generalization of the classical fluid theory. Extrusions of polymer fluids, cooling of metallic plates in a bath, rotating machinery, and so forth are the applications of couple stress fluids. Sajid et al. [7] provided the study on some two-dimensional flows with couple stresses; Malashetty et al. [8] investigated the double diffusive convection in a couple stress fluid saturated porous layer with Soret effect; melting heat transfer in the boundary layer flow of a couple stress fluid over a stretching surface is considered by Hayat et al. [9]; Khan and colleagues found the approximate solution of the couple stress fluid between expanding and contracting walls [10]; an analysis has been provided for three-dimensional magnetohydrodynamics flow of couple stress fluid with Newtonian heating by Ramzan et al. [11].

Blunt rotating bodies moving in a fluid produce the forced flow over rotating bodies in which stagnation region experiences the highest pressure, high heat transfer, and so forth, making it significant to consider it as a research problem. Hannah [12] was the first to examine the forced flow and explain the axisymmetric stagnation point flow towards a rotating disk. Wang [13] investigated the nonaligned stagnation point flow towards a rotating disk and found that the flow configuration became complicated by nonalignment. Dinarvand [14] reevaluated the Wang [13] results by employing the homotopy analytical approach.

Homotopy analytical approach presented by Liao [15] is the most effective technique to solve nonlinear equations as it provides wide flexibility to choose the convergence region of the obtained series solution with the help of $\hbar$, "the convergence control parameter." The technique is a powerful tool to acquire the analytical solution of the problem which 
converges relatively close to the exact solutions. Many investigations have been made [16] to scrutinize its convergence and found it relatively convenient as compared to other techniques.

In present study, the couple stress fluid has been investigated for the stagnation flow towards the off-centered rotating disk. The current investigation is an extension in the previous work of Wang [13] with the replacement of viscous fluid to couple stress fluid. The three-dimensional partial differential equations with body stresses and body couples are transformed to ordinary differential equations with the use of similarity transformation. The homotopy analysis method is employed to obtain the analytic solution of the governing problem in the form of series. The convergence analysis has also been done to determine the convergence region of the achieved solution. The influences of pertinent parameters on the velocity profiles are examined through graphs. The considered problem will recover the problem of Wang [13] as a special case.

\section{Problem Formulation}

Consider the steady, incompressible, and off-centered stagnation point flow of a couple stress fluid over an infinite disk rotating with angular velocity $\Omega$. The stagnation flow is at distance $b$ along $z$-axis intruding on a rotating disk (shown in Figure 1). Let $u, v$, and $w$ be the velocity components along $x, y$ and $z$ directions, respectively. The partial differential equation governing the problem can be described as

$$
\nabla \cdot V=0
$$

$$
\rho\left(\frac{\partial V}{\partial t}+(V \cdot \nabla) V\right)=-\nabla p+\mu \nabla^{2} V-\gamma \nabla^{4} V
$$

where $\nabla^{2}=\left(\partial^{2} / \partial x^{2}\right)+\left(\partial^{2} / \partial y^{2}\right)+\left(\partial^{2} / \partial z^{2}\right)$ and $\nabla^{4}=$ $\left(\partial^{4} / \partial x^{4}\right)+\left(\partial^{4} / \partial y^{4}\right)+\left(\partial^{4} / \partial z^{4}\right)+2\left(\partial^{4} / \partial x^{2} \partial y^{2}\right)+2\left(\partial^{4} / \partial y^{2} \partial z^{2}\right)+$ $2\left(\partial^{4} / \partial x^{2} \partial z^{2}\right)$ subject to the boundary conditions near the disk are

$$
u=-\Omega y, \quad v=\Omega(x-b), \quad w=0,
$$

and at infinity

$$
u=a x, \quad v=a y, \quad w=-2 a z,
$$

where $a$ represents the strength of the stagnation flow.

The similarity transformations presented by Wang [13] are

$$
\begin{gathered}
u=a x f^{\prime}(\eta)-\Omega y g(\eta)+b \Omega k(\eta), \\
v=a y f^{\prime}(\eta)+\Omega x g(\eta)+b \Omega h(\eta), \\
w=-2 \sqrt{a v} f(\eta),
\end{gathered}
$$

where $v$ is the kinematic viscosity and

$$
\eta=z \sqrt{\frac{a}{v}}
$$



FIGURE 1: Off-centered stagnation flow on a rotating disk.

Using the above similarity transformations in (1), the equation of continuity is satisfied whereas the governing partial differential equation reduced to the following equations:

$$
\begin{gathered}
f^{\prime \prime \prime}-f^{\prime 2}+\alpha^{2} g^{2}+2 f f^{\prime}-\lambda f^{\prime \prime \prime \prime \prime}+1=0, \\
g^{\prime \prime}-2 g f^{\prime}+2 f g^{\prime}-\lambda g^{\prime \prime \prime \prime}=0, \\
k^{\prime \prime}-k f^{\prime}+\alpha h g+2 f k^{\prime}-\lambda k^{\prime \prime \prime \prime}=0, \\
h^{\prime \prime}-\alpha g k-h f^{\prime}+2 f h^{\prime}-\lambda h^{\prime \prime \prime \prime}=0,
\end{gathered}
$$

where $\alpha=\Omega / a$ is nondimensional rotational parameter and $\lambda=a \gamma / \nu^{2} \rho$ is the non-dimensional couple stress parameter. The boundary conditions take the form

$$
\begin{aligned}
f(0)=0, & f^{\prime}(0)=0, \quad f^{\prime}(\infty)=1, \\
g(0)=1, & g(\infty)=0, \\
k(0)=0, & k(\infty)=0, \\
h(0)=1, & h(\infty)=0,
\end{aligned}
$$

where primes denote the differentiation with respect to $\eta$.

Equations (7) represent the system of couple stress equations. However, if the value of couple stress parameter $\lambda$ is set to zero in the presented system, (7) will be reduced to the Navier-Stokes equations of stagnation flow [13].

The pressure $p$ can be recovered by

$$
p=p_{0}-\frac{1}{2} \rho a^{2}\left(x^{2}+y^{2}\right)-\frac{1}{2} \rho\left(w^{2}-2 \nu w_{z}+2 \gamma^{\prime} w_{z z z}\right),
$$

where $p_{0}$ is the pressure at the origin and $\rho$ is the fluid density. The shear stress on the disk for the couple stress fluid is given by

$$
\begin{aligned}
\tau_{x}= & \left.\left(\rho \nu \frac{\partial u}{\partial z}-\gamma \frac{\partial^{3} u}{\partial z^{3}}\right)\right|_{z=0} \\
= & \rho a \sqrt{v a}\left[x f^{\prime \prime}(0)-\alpha y g^{\prime}(0)+b \alpha k^{\prime}(0)\right] \\
& +\frac{a^{2} \sqrt{a} \gamma}{\nu^{3 / 2}}\left[-x f^{\prime \prime \prime \prime}(0)+\alpha y g^{\prime \prime \prime}(0)-b \alpha k^{\prime \prime \prime}(0)\right],
\end{aligned}
$$




$$
\begin{aligned}
\tau_{y}= & \left.\left(\rho \nu \frac{\partial v}{\partial z}-\gamma \frac{\partial^{3} v}{\partial z^{3}}\right)\right|_{z=0} \\
= & \rho a \sqrt{v a}\left[y f^{\prime \prime}(0)+\alpha x g^{\prime}(0)+b \alpha h^{\prime}(0)\right] \\
& -\frac{a^{2} \sqrt{a} \gamma}{v^{3 / 2}}\left[y f^{\prime \prime \prime \prime}(0)+\alpha x g^{\prime \prime \prime}(0)+b \alpha k^{\prime \prime \prime}(0)\right] .
\end{aligned}
$$

The shear centre can be obtained by setting (10) to zero and solving for $(x, y)$ as the shear stress is zero at the centre. The torque experienced by the disk of radius $R$ is

$$
M=\int_{0}^{R} \int_{0}^{2 \pi}\left(\tau_{y} \cos \theta-\tau_{x} \sin \theta\right) r^{2} d \theta d r
$$

where $(r, \theta)$ are cylindrical coordinates. Since $x=r \cos \theta-b$ and $y=r \sin \theta$, we find the torque as

$$
M=\frac{\pi}{2} \frac{a \sqrt{a}}{v^{3 / 2}} \alpha R^{4}\left(\nu^{2} \rho g^{\prime}(0)-a \gamma g^{\prime \prime \prime}(0)\right),
$$

which is unaltered by the non-aligned disk axis and flow axis.

\section{Analytical Approximations by means of HAM}

The auxiliary linear operators, $\mathscr{L}_{1}[f]$ and $\mathscr{L}_{2}[g, k, h]$, are selected for (7):

$$
\mathscr{L}_{1}[f]=\frac{d^{3}}{d \eta^{3}}-\frac{d}{d \eta}, \quad \mathscr{L}_{2}[g, h, k]=\frac{d^{2}}{d \eta^{2}}-1,
$$

and also the initial guesses satisfying the initial conditions in (8) are

$$
\begin{array}{cc}
f_{0}(\eta)=\eta-1+e^{-\eta}, & g_{0}(\eta)=e^{-\eta}, \\
k_{0}(\eta)=\eta e^{-\eta}, & h_{0}(\eta)=e^{-\eta}
\end{array}
$$

satisfying the following properties:

$$
\mathscr{L}_{1}\left[c_{1}+c_{2} e^{\eta}+c_{3} e^{-\eta}\right]=0, \quad \mathscr{L}_{2}\left[c_{4} e^{\eta}+c_{5} e^{-\eta}\right]=0 .
$$

3.1. Zeroth Order Deformation Problems. The problems at the zeroth order are given by

$$
\begin{gathered}
(1-p) \mathscr{L}_{1}\left[\tilde{f}(\eta, p)-f_{0}(\eta)\right]=p \hbar N_{1}[\tilde{f}(\eta, p), \tilde{g}(\eta, p)], \\
(1-p) \mathscr{L}_{2}\left[\tilde{g}(\eta, p)-g_{0}(\eta)\right]=p \hbar N_{2}[\tilde{f}(\eta, p), \widetilde{g}(\eta, p)], \\
(1-p) \mathscr{L}_{2}\left[\widetilde{k}(\eta, p)-k_{0}(\eta)\right] \\
=p \hbar N_{3}[\tilde{f}(\eta, p), \tilde{g}(\eta, p), \tilde{k}(\eta, p), \widetilde{h}(\eta, p)], \\
(1-p) \mathscr{L}_{2}\left[\tilde{h}(\eta, p)-h_{0}(\eta)\right] \\
=p \hbar N_{4}[\tilde{f}(\eta, p), \tilde{g}(\eta, p), \tilde{k}(\eta, p), \widetilde{h}(\eta, p)],
\end{gathered}
$$

where

$$
\begin{aligned}
& N_{1}[\tilde{f}(\eta, p), \tilde{g}(\eta, p)] \\
& =\frac{\partial^{3} \tilde{f}(\eta, p)}{\partial \eta^{3}}-\left(\frac{\partial \tilde{f}(\eta, p)}{\partial \eta}\right)^{2}+\alpha^{2}(\tilde{g}(\eta, p))^{2} \\
& +2 \tilde{f}(\eta, p) \frac{\partial^{2} \tilde{f}(\eta, p)}{\partial \eta^{2}}-\lambda \frac{\partial^{5} \tilde{f}(\eta, p)}{\partial \eta^{5}}+1, \\
& N_{2}[\tilde{f}(\eta, p), \tilde{g}(\eta, p)] \\
& =\frac{\partial^{2} \tilde{g}(\eta, p)}{\partial \eta^{2}}-2 \widetilde{g}(\eta, p) \frac{\partial \tilde{f}(\eta, p)}{\partial \eta} \\
& +2 \tilde{f}(\eta, p) \frac{\partial \widetilde{g}(\eta, p)}{\partial \eta}-\lambda \frac{\partial^{4} \tilde{g}(\eta, p)}{\partial \eta^{4}}, \\
& N_{3}[\tilde{f}(\eta, p), \widetilde{g}(\eta, p), \widetilde{k}(\eta, p), \widetilde{h}(\eta, p)] \\
& =\frac{\partial^{2} \widetilde{k}(\eta, p)}{\partial \eta^{2}}-\tilde{k}(\eta, p) \frac{\partial \tilde{f}(\eta, p)}{\partial \eta} \\
& +\alpha \tilde{h}(\eta, p) \tilde{g}(\eta, p)+2 \tilde{f}(\eta, p) \frac{\partial \tilde{k}(\eta, p)}{\partial \eta} \\
& -\lambda \frac{\partial^{4} \tilde{k}(\eta, p)}{\partial \eta^{4}} \\
& N_{4}[\tilde{f}(\eta, p), \tilde{g}(\eta, p), \tilde{k}(\eta, p), \tilde{h}(\eta, p)] \\
& =\frac{\partial^{2} \widetilde{h}(\eta, p)}{\partial \eta^{2}}-\alpha \widetilde{g}(\eta, p) \tilde{k}(\eta, p) \\
& -\tilde{h}(\eta, p) \frac{\partial \tilde{f}(\eta, p)}{\partial \eta}+2 \tilde{f}(\eta, p) \frac{\partial \widetilde{h}(\eta, p)}{\partial \eta} \\
& -\lambda \frac{\partial^{4} \tilde{h}(\eta, p)}{\partial \eta^{4}}
\end{aligned}
$$

in which $p \in[0,1]$ is the embedding parameter and $\hbar$ is the auxiliary nonzero parameter. By Taylor's theorem,

$$
\begin{aligned}
& \tilde{f}(\eta, p)=f_{0}(\eta)+\sum_{m=1}^{\infty} f_{m}(\eta) p^{m}, \\
& \tilde{g}(\eta, p)=g_{0}(\eta)+\sum_{m=1}^{\infty} g_{m}(\eta) p^{m}, \\
& \tilde{k}(\eta, p)=k_{0}(\eta)+\sum_{m=1}^{\infty} k_{m}(\eta) p^{m}, \\
& \tilde{h}(\eta, p)=h_{0}(\eta)+\sum_{m=1}^{\infty} h_{m}(\eta) p^{m} .
\end{aligned}
$$


3.2. mth Order Deformation Problems. The general HAM equation for mth order can be given by

$$
\begin{aligned}
& \mathscr{L}_{1}\left[f_{m}(\eta)-\chi_{m} f_{m-1}(\eta)\right]=\hbar R_{1, m}(\eta), \\
& \mathscr{L}_{2}\left[g_{m}(\eta)-\chi_{m} g_{m-1}(\eta)\right]=\hbar R_{2}(\eta), \\
& \mathscr{L}_{2}\left[k_{m}(\eta)-\chi_{m} k_{m-1}(\eta)\right]=\hbar R_{3, m}(\eta), \\
& \mathscr{L}_{2}\left[h_{m}(\eta)-\chi_{m} h_{m-1}(\eta)\right]=\hbar R_{4, m}(\eta)
\end{aligned}
$$

with the following boundary conditions:

$$
\begin{gathered}
f_{m}(0)=0, \quad f_{m}^{\prime}(0)=0, \quad f_{m}^{\prime}(\infty)=0 \\
g_{m}(0)=0, \quad g_{m}(\infty)=0 \\
k_{m}(0)=0, \quad k_{m}(\infty)=0 \\
h_{m}(0)=0, \quad h_{m}(\infty)=0, \\
\chi_{m}= \begin{cases}0, & m \leq 1 \\
1, & m \geq 2\end{cases}
\end{gathered}
$$

$$
\begin{aligned}
& R_{1, m}(\eta) \\
& =f_{m-1}^{\prime \prime \prime}(\eta) \\
& \quad+\sum_{i=0}^{m-1}\left(-f_{i}^{\prime}(\eta) f_{m-1-i}^{\prime}(\eta)+\alpha g_{i}(\eta) g_{m-1-i}(\eta)\right. \\
& \left.\quad+2 f_{i}(\eta) f_{m-1-i}^{\prime \prime}(\eta)\right)-\lambda f_{m-1}^{\prime \prime \prime \prime \prime}(\eta)+\left(1-\chi_{m}\right),
\end{aligned}
$$

$$
\begin{aligned}
& R_{2, m}(\eta) \\
& =g_{m-1}^{\prime \prime}(\eta) \\
& \quad+\sum_{i=0}^{m-1}\left(-2 g_{i}(\eta) f_{m-1-i}^{\prime}(\eta)+2 f_{i}(\eta) g_{m-1-i}^{\prime}(\eta)\right) \\
& \quad-\lambda g_{m-1}^{\prime \prime \prime \prime}(\eta), \\
& R_{3, m}(\eta) \\
& =k_{m-1}^{\prime \prime}(\eta) \\
& \quad+\sum_{i=0}^{m-1}\left(-k_{i}(\eta) f_{m-1-i}^{\prime}(\eta)+\alpha h_{i}(\eta) g_{m-1-i}(\eta)\right. \\
& R_{4, m}(\eta) \\
& =h_{m-1}^{\prime \prime}(\eta) \\
& \quad+\sum_{i=0}^{m-1}\left(-\alpha k_{i}(\eta) g_{m-1-i}(\eta)-h_{i}(\eta) f_{m-1-i}^{\prime}(\eta)\right. \\
& \left.+2 f_{i}(\eta) h_{m-1-i}^{\prime}(\eta)\right)-\lambda h_{m-1}^{\prime \prime \prime \prime}(\eta) .
\end{aligned}
$$

\section{Conclusion}

The present paper modelled the nonaligned flow of a couple stress fluid towards a rotating disk. The governing equations transformed to ordinary differential equations with the help of suitable similarity transformation. The analytical
According to the above-defined method, the linear equations (19) with the boundary conditions (20) in the order $m=$ $1,2,3, \ldots$ can be solved easily by means of any computational software, such as Mathematica/MATLAB.

\section{Results and Discussion}

In this paper, the effect of couple stress fluid on steady stagnation point flow over a rotating disk has been examined. The system of nonlinear ordinary differential equations (7) under the boundary conditions (8) has been solved analytically by employing HAM. The physical aspects of the considered problem have been examined for the radial velocity $f^{\prime}(\eta)$, azimuthal velocity $g(\eta)$, and induced velocity functions $k(\eta)$ and $h(\eta)$ that arise due to the nonalignment in the flow axis and rotating disk axis in account of rotational parameter $\alpha$ and couple stress parameter $\lambda$.

To ensure the convergence of HAM for the obtained solutions, it is necessary to find the appropriate value of $\hbar$ for which the results converge to the exact solution. In Figures 2 and 3 the $\hbar$ graphs are computed for $f^{\prime \prime \prime}(0), g^{\prime \prime}(0), k^{\prime \prime}(0)$, and $h^{\prime \prime}(0)$ to achieve the convergence region and the admissible range of values for different values of rotational and couple stress parameters.

Figure 4 illustrated the profile of radial velocity $f^{\prime}(\eta)$ against the rotational parameter $\alpha$. It can be seen that there is an increase in the radial velocity with the increase in $\alpha$, high rotational parameter but as the flow approaches infinity it all converges at unity. The effect of couple stress parameter $\lambda$ on the radial velocity $f^{\prime}(\eta)$ has been noticed in Figure 5. With the rotation in the disk and increasing couple stresses the flow velocity also increases. For $\lambda=0$ the flow retains the Newtonian fluid.

The insight of azimuthal velocity $g(\eta)$ can be perceived in Figures 6 and 7. The influence of increasing rotation in the disk results in the decrease in flow velocity in azimuthal direction (see Figure 6). Also increase in the magnitude of couple stress parameter $\lambda$ causes reduction in the azimuthal velocity $g(\eta)$ as shown in Figure 7.

In Figure 8, the induced velocity function $k(\eta)$ shows the rapid rise and then exponential decay in the fluid flow as the rotational parameter $\alpha$ increases. A similar behavior is observed in Figure 9 for velocity function $k(\eta)$ with the varying values of couple stress parameter $\lambda$. It can be depicted in Figure 10 that the induced velocity function $h(\eta)$ falls, decreasing the boundary wall thickness with the increase in rotational parameter $\alpha$. Figure 11 demonstrates the induced velocity profile of $h(\eta)$ for different values of $\lambda$. It reveals that the presence of stresses in the fluid does not support the offcentered velocity function. and also a rapid increment in velocity has been observed with 


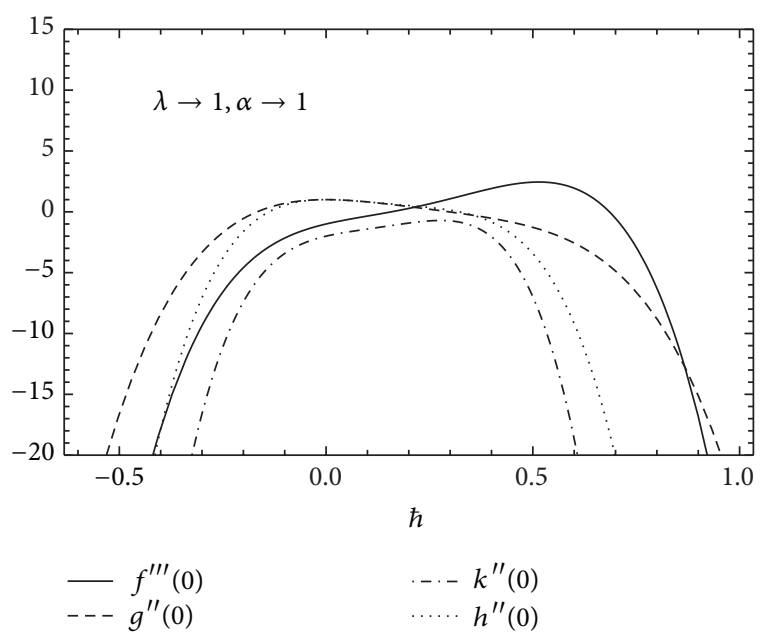

Figure 2: The $\hbar$ curves for $f^{\prime \prime \prime}(0), g^{\prime \prime}(0), k^{\prime \prime}(0)$, and $h^{\prime \prime}(0)$.



Figure 3: The $\hbar$ curves for $f^{\prime \prime \prime}(0), g^{\prime \prime}(0), k^{\prime \prime}(0)$, and $h^{\prime \prime}(0)$.

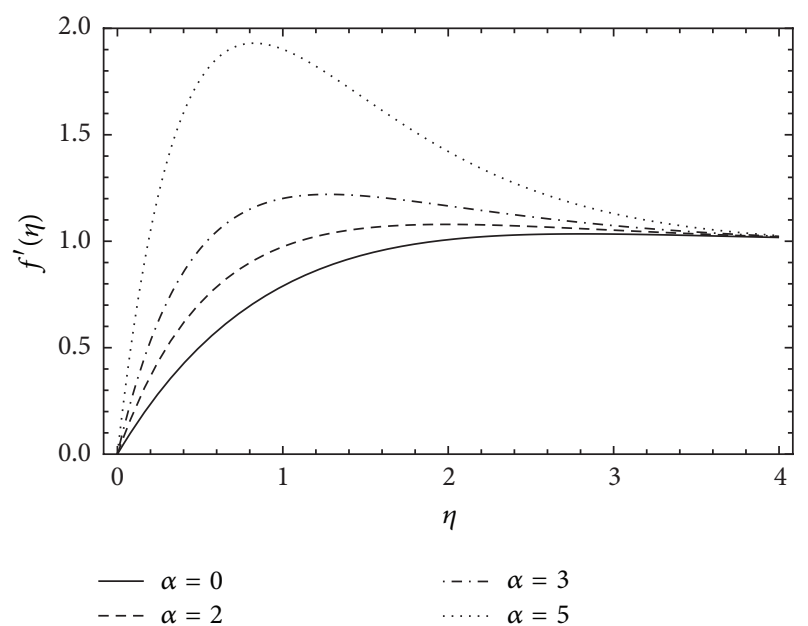

FIGURE 4: The influence of the rotation parameter $\alpha$ on $f^{\prime}(\eta)$ for $\lambda=1, \hbar=-0.1$.

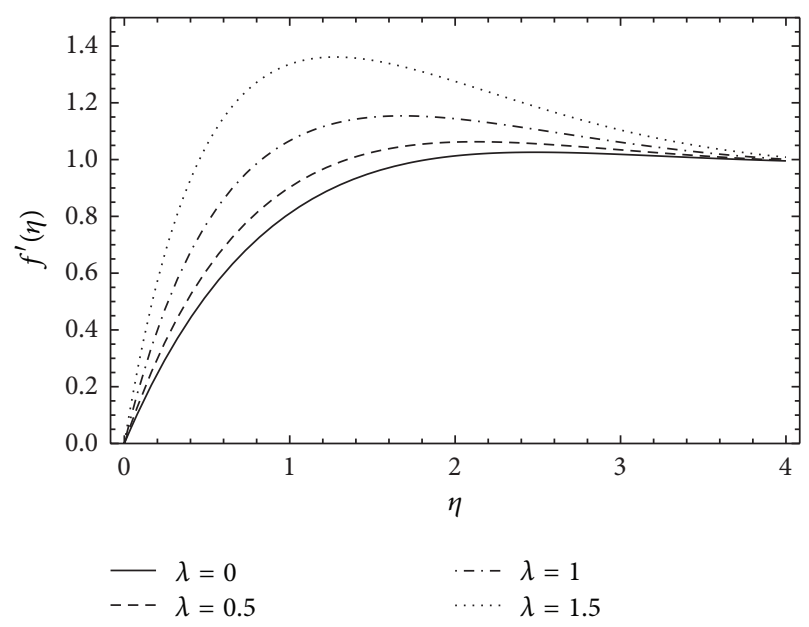

FIgURE 5: The influence of the couple stress parameter $\lambda$ on $f^{\prime}(\eta)$ for $\alpha=1, \hbar=-0.2$, and $\lambda=0$ (Newtonian case).

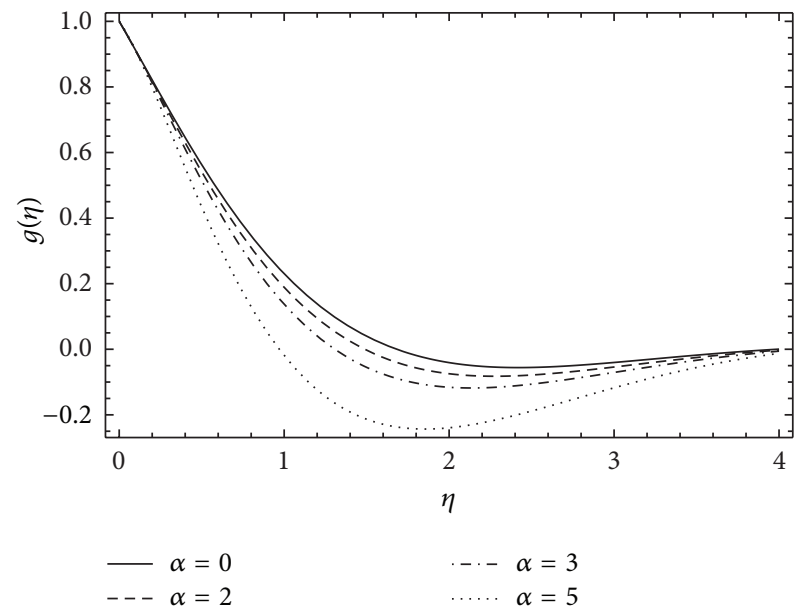

FIGURE 6: The influence of the rotation parameter $\alpha$ on $g(\eta)$ for $\lambda=$ $1, \hbar=-0.2$

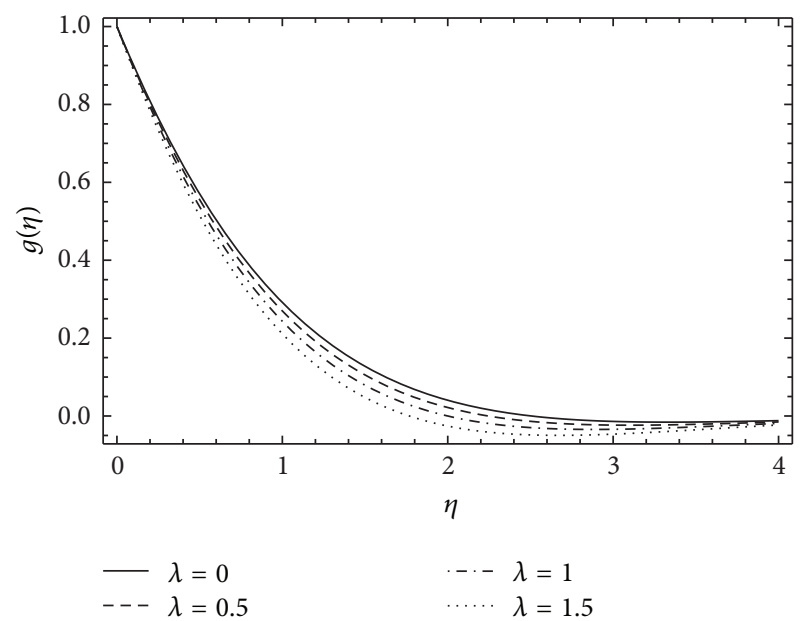

FIGURE 7: The influence of the couple stress parameter $\lambda$ on $g(\eta)$ for $\alpha=2, \hbar=-0.1$, and $\lambda=0$ (Newtonian case). 


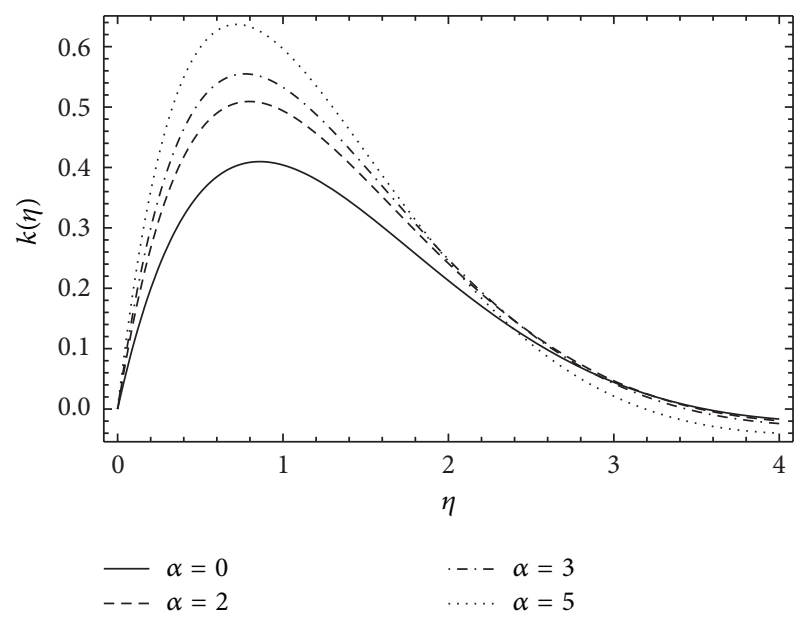

FIGURE 8: The influence of the rotation parameter $\alpha$ on $k(\eta)$ for $\lambda=$ $1, \hbar=-0.1$.

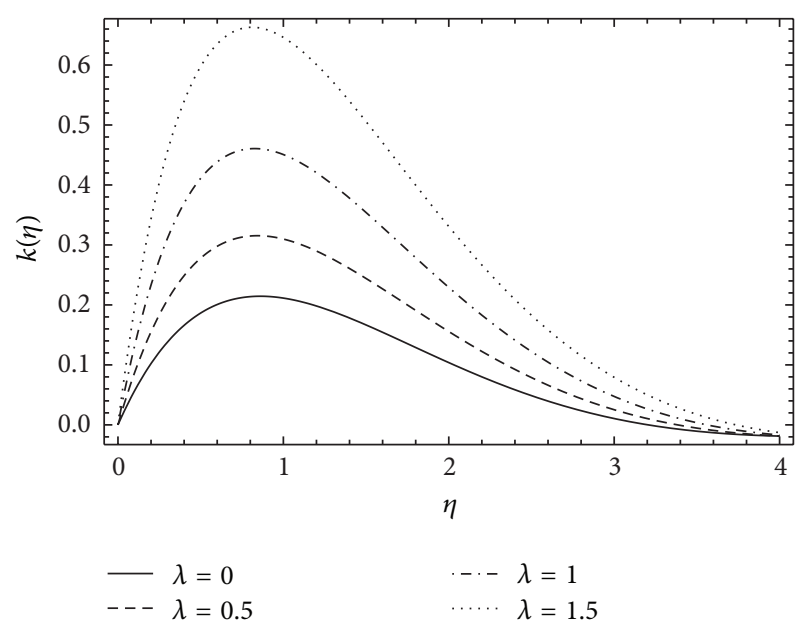

FIGURE 9: The influence of the couple stress parameter $\lambda$ on $k(\eta)$ for $\alpha=1, \hbar=-0.1$, and $\lambda=0$ (Newtonian case).

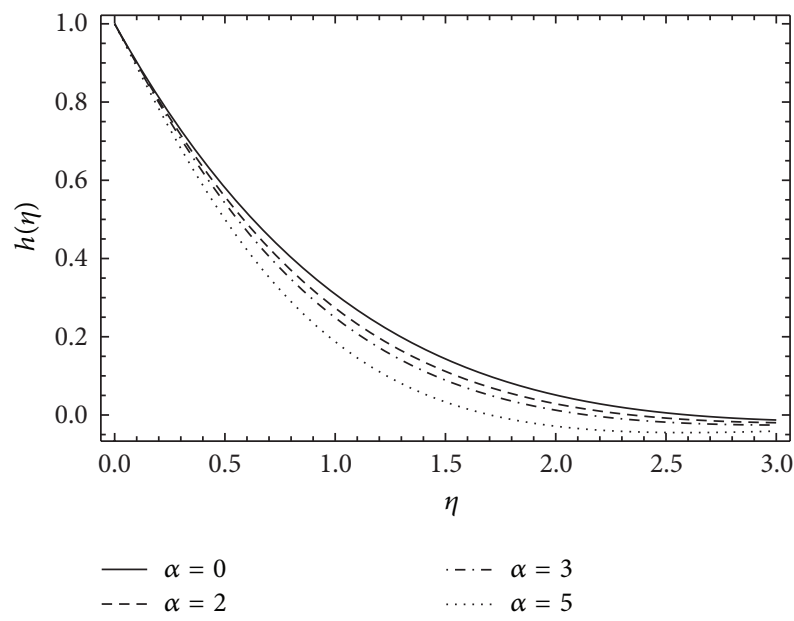

FIGURE 10: The influence of the rotation parameter $\alpha$ on $h(\eta)$ for $\lambda=1, \hbar=-0.1$.

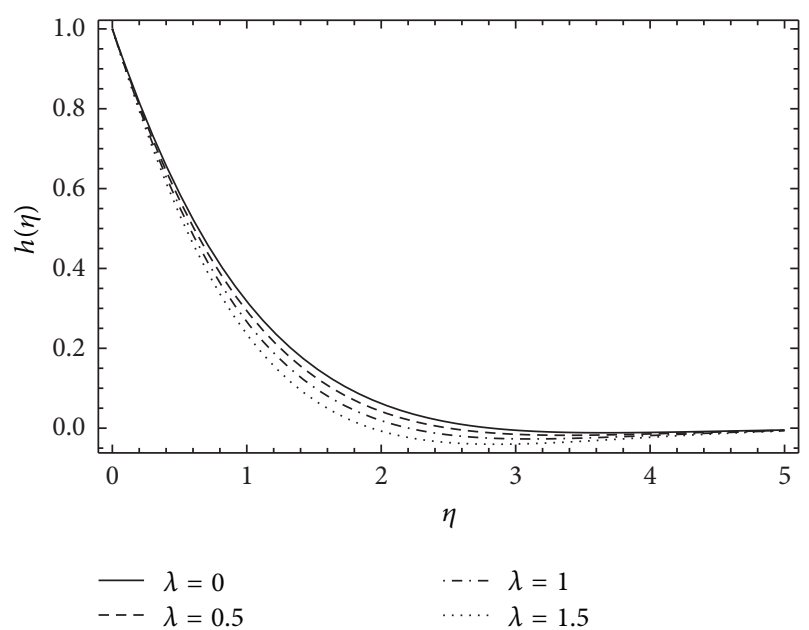

FIGURE 11: The influence of the couple stress parameter $\lambda$ on $h(\eta)$ for $\alpha=1, \hbar=-0.1$, and $\lambda=0$ (Newtonian case).

computation has been carried out by the homotopy analysis method and the influences of the pertinent parameters are investigated on the velocity profiles of the fluid in radial, azimuthal, and off-centered directions. Present investigation reveals that the couple stress parameter $\lambda$ and disk rotational parameter $\alpha$ have the same effect of increasing radial velocity and decreasing the remaining velocity functions with the increase in respective parameters. It can be concluded from the above study that the rotation in the fluid particles and rotation in the disk give the combined effect of rotation in the fluid. Also, the equations for the shear stress and torque experienced by the disk have been evaluated for the couple stress flow and it was found that the torque remains unchanged with the off-centered flow.

\section{Notations}

$(x, y, z):$ Space coordinates

$(u, v, w)$ : Velocity components

$\Omega: \quad$ Angular velocity

$\rho: \quad$ Density

$\mu: \quad$ Viscosity

$v$ : Kinematic viscosity

a: $\quad$ Strength of flow

$\gamma: \quad$ Couple stress parameter

$b$ : $\quad$ Distance between disk axis and flow axis

$\alpha: \quad$ Nondimensional rotational parameter

$\lambda: \quad$ Dimensionless couple stress parameter

$(r, \theta): \quad$ Cylindrical coordinates

$p: \quad$ Pressure of the fluid

$R: \quad$ Radius of the disk

$M: \quad$ Torque experienced by disk

$\tau_{x}, \tau_{y}: \quad$ Components of shear stress.

\section{Conflict of Interests}

The authors declare that there is no conflict of interests regarding the publication of this paper. 


\section{Acknowledgment}

The author Najeeb Alam Khan is highly thankful and grateful to the Dean Faculty of Sciences, University of Karachi, Karachi 75270, Pakistan for supporting and facilitating this research work.

\section{References}

[1] T. Von Kármán, "Uber laminare und turbulente Reibung," Zeitschrift für Angewandte Mathematik und Mechanik, vol. 1, no. 4, pp. 233-252, 1921.

[2] C. Ming, L. Zheng, and X. Zhang, "Steady flow and heat transfer of the power-law fluid over a rotating disk," International Communications in Heat and Mass Transfer, vol. 38, no. 3, pp. 280284, 2011

[3] M. Turkyilmazoglu, "Purely analytic solutions of magnetohydrodynamic swirling boundary layer flow over a porous rotating disk," Computers \& Fluids, vol. 39, no. 5, pp. 793-799, 2010.

[4] M. M. Hashmi, T. Hayat, and A. Alsaedi, "On the analytic solutions for squeezing flow of nanofluid between parallel disks," Nonlinear Analysis; Modelling and Control, vol. 17, no. 4, pp. 418430, 2012.

[5] A. Ahmadpour and K. Sadeghy, "Swirling flow of Bingham fluids above a rotating disk: an exact solution," Journal of NonNewtonian Fluid Mechanics, vol. 197, pp. 41-47, 2013.

[6] V. K. Stokes, "Couple stresses in fluids," Physics of Fluids, vol. 9, no. 9, pp. 1709-1715, 1966.

[7] M. Sajid, N. Ali, Z. Abbas, T. Javed, and M. Imran, "Some twodimensional flows with couple stresses," Journal of Engineering Physics and Thermophysics, vol. 85, no. 3, pp. 649-654, 2012.

[8] M. S. Malashetty, I. Pop, P. Kollur, and W. Sidram, "Soret effect on double diffusive convection in a Darcy porous medium saturated with a couple stress fluid," International Journal of Thermal Sciences, vol. 53, pp. 130-140, 2012.

[9] T. Hayat, M. Mustafa, Z. Iqbal, and A. Alsaedi, "Stagnationpoint flow of couple stress fluid with melting heat transfer," Applied Mathematics and Mechanics, vol. 34, no. 2, pp. 167-176, 2013.

[10] N. A. Khan, A. Mahmood, and A. Ara, "Approximate solution of couple stress fluid with expanding or contracting porous channel," Engineering Computation, vol. 30, no. 3, pp. 399-408, 2013.

[11] M. Ramzan, M. Farooq, A. Alsaedi, and T. Hayat, "MHD threedimensional flow of couple stress fluid with Newtonian heating," The European Physical Journal Plus, vol. 128, article 49, 2013.

[12] D. M. Hannah, "Forced flow against a rotating disc, British Aeronautical Research Council Reports and Memoranda," Tech. Rep. 2772, University of Michigan, 1947.

[13] C. Y. Wang, "Off-centered stagnation flow towards a rotating disc," International Journal of Engineering Science, vol. 46, no. 4, pp. 391-396, 2008.

[14] S. Dinarvand, "On explicit, purely analytic solutions of offcentered stagnation flow towards a rotating disc by means of HAM," Nonlinear Analysis; Real World Applications, vol. 11, no. 5, pp. 3389-3398, 2010.

[15] S. J. Liao, The proposed homotopy analysis technique for the solution of nonlinear problems [Ph.D. thesis], Shanghai Jiao Tong University, 1992.
[16] Z. M. Odibat, "A study on the convergence of homotopy analysis method," Applied Mathematics and Computation, vol. 217, no. 2, pp. 782-789, 2010. 

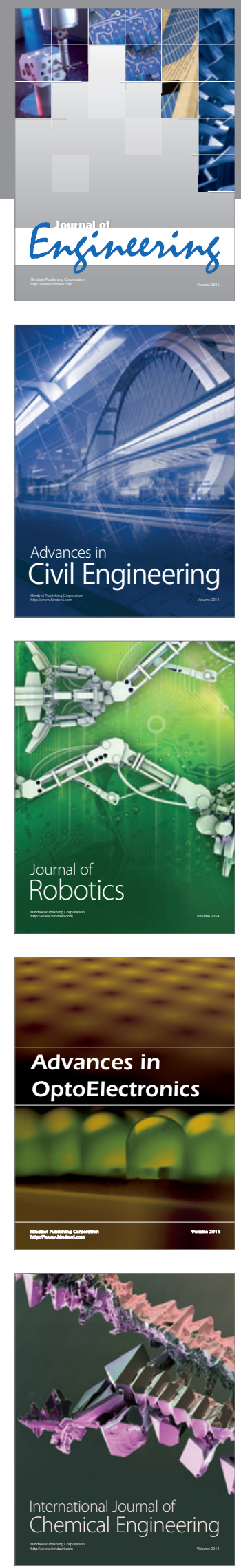

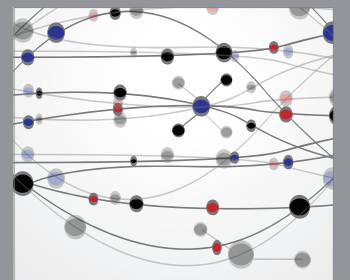

The Scientific World Journal
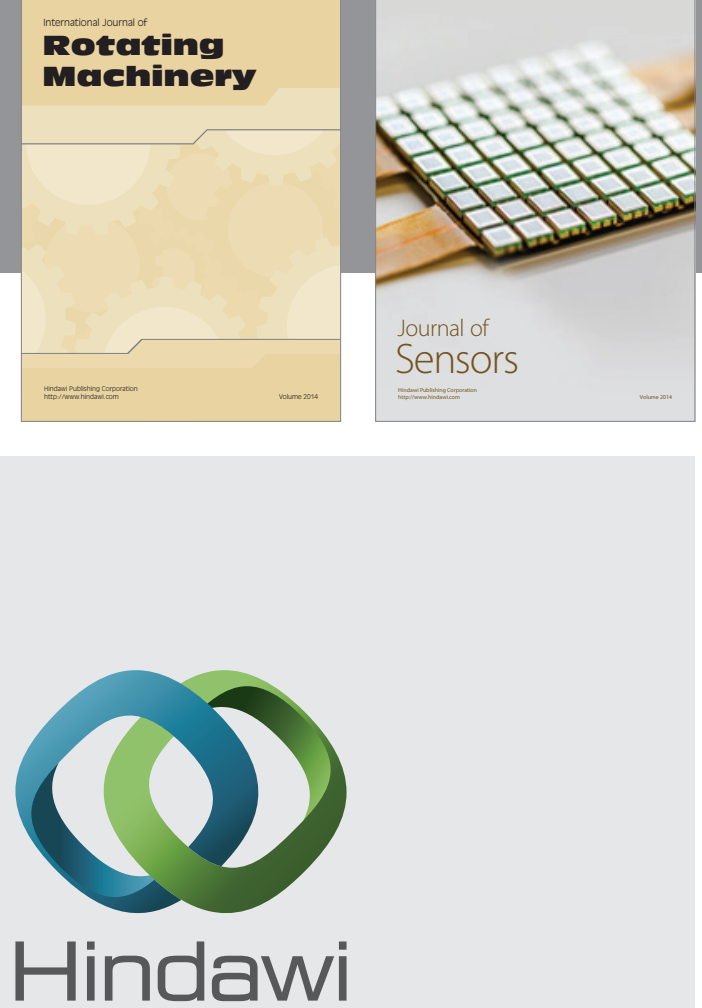

Submit your manuscripts at http://www.hindawi.com
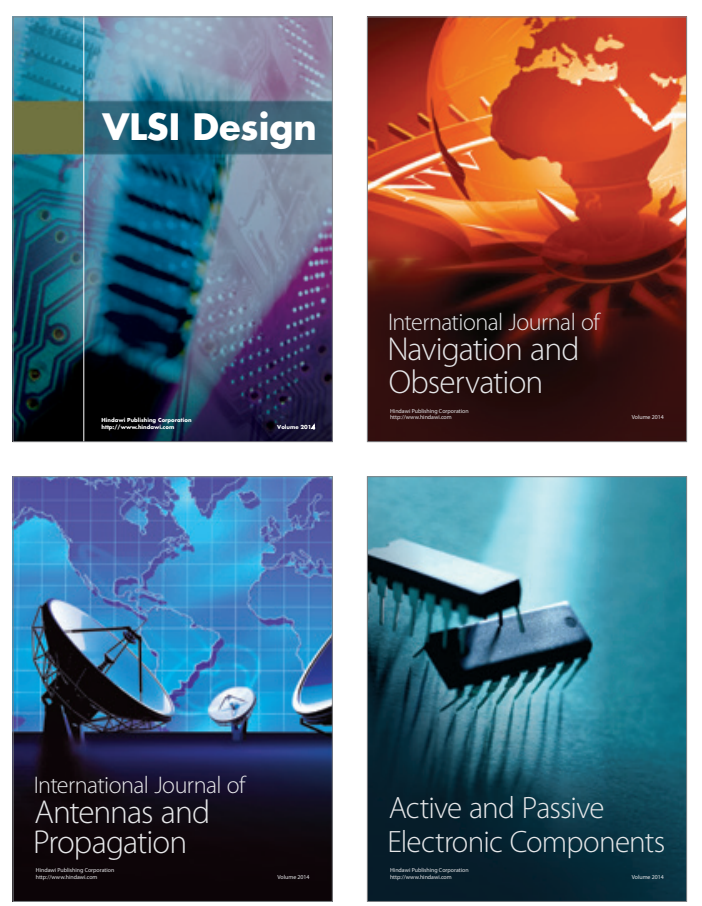
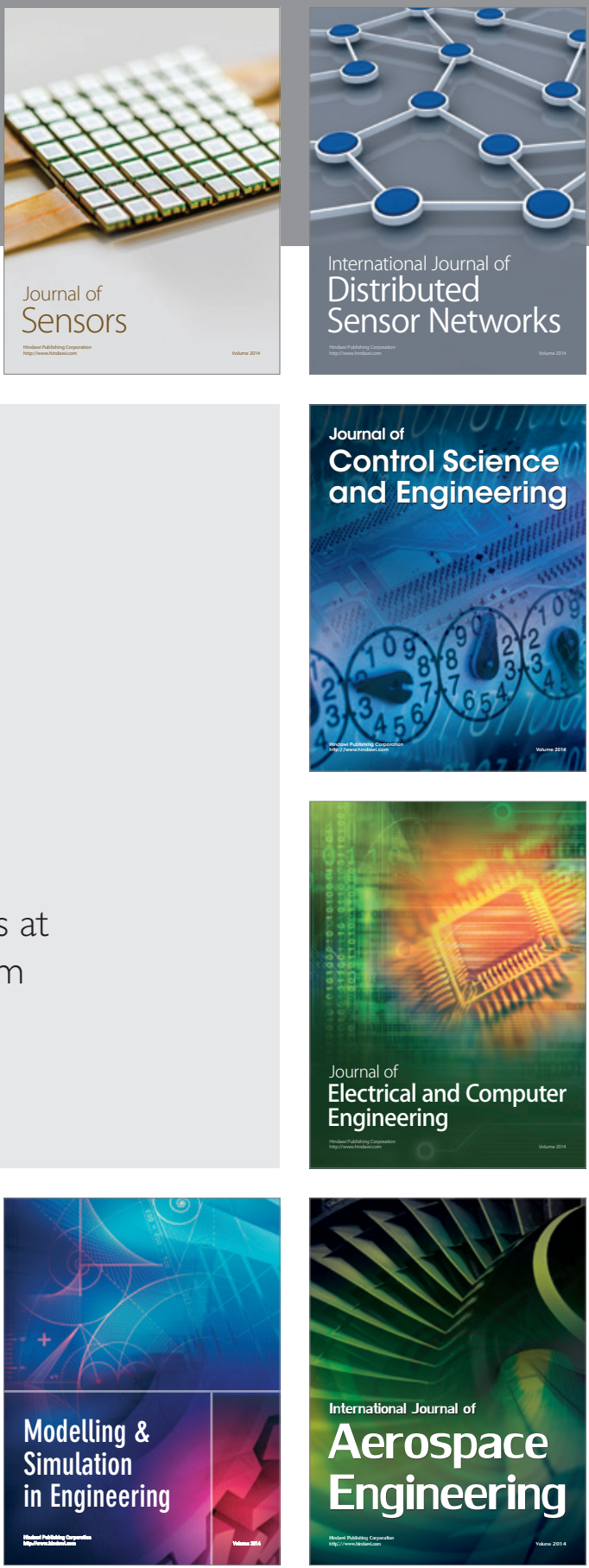

Journal of

Control Science

and Engineering
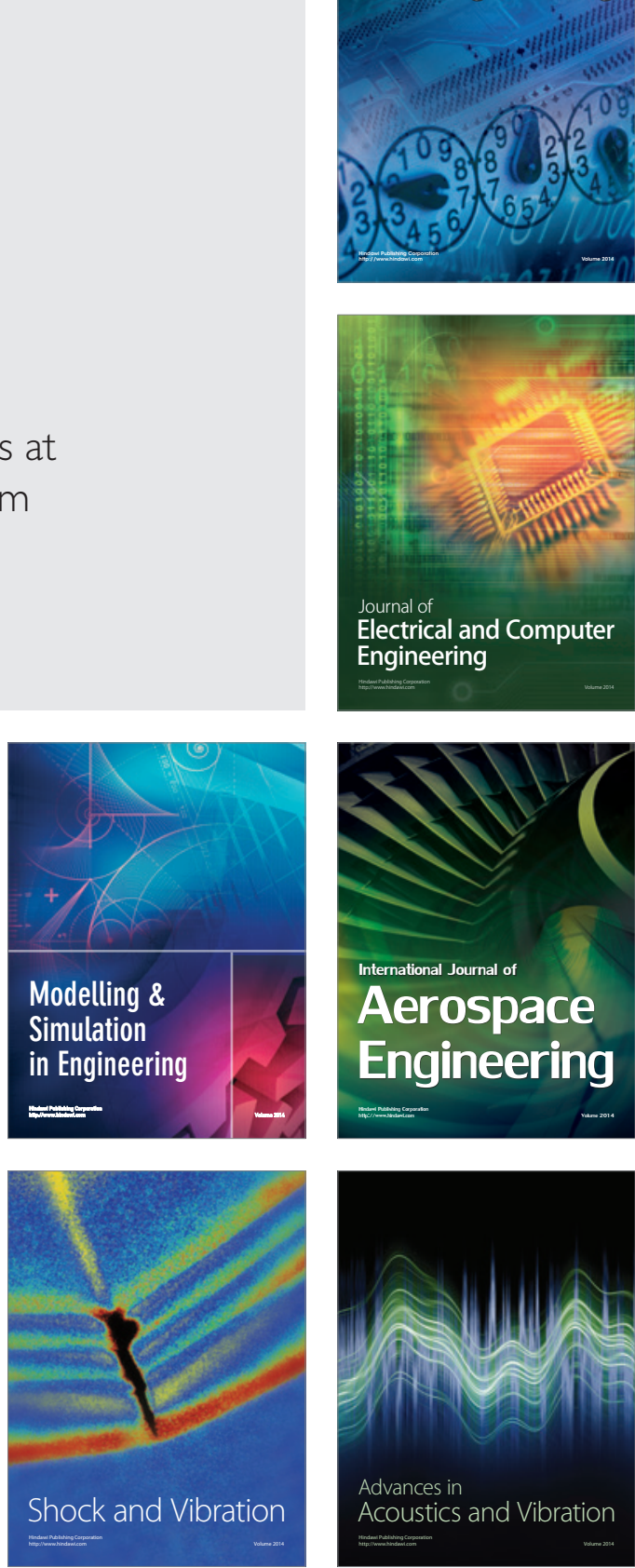